\title{
NUMERICAL COMPUTATIONS FOR ANTIPLANE SHEAR IN A GRANULAR FLOW MODEL
}

\author{
BY \\ XABIER GARAIZAR \\ North Carolina State University, Raleigh, NC
}

\begin{abstract}
We describe an algorithm for the numerical resolution of elasto-plastic deformations in the context of antiplane shear models. The algorithm is a secondorder Godunov method. For these models the eigenvalues associated to the hyperbolic system are discontinuous. We test the algorithm on several examples.

We also describe an algorithm for the resolution of the corresponding Riemann problems.
\end{abstract}

1. Introduction. The deformations of materials are described by a system of partial differential equations. Some of these equations, constitutive laws, are particular to the material and describe the reaction of the material to changes on the stress. For elasto-plastic materials, the deformation could be reversible (elastic) or irreversible (plastic). Which of the two types of deformation occurs is determined by the past history of the material point and whether the material is loading (an appropriate measure of total stress is increasing) or unloading (the total stress is decreasing). The two possible descriptions of the deformation can be viewed as a change in the constitutive laws when the total stress reaches a maximum value determined by the past history of the material. We refer to this maximum value as the yield stress. As we will show later, the change in the constitutive laws introduces some difficulties in the numerical resolution of elasto-plastic deformations.

The model we consider is a one-dimensional simplification of the antiplane shear model presented by Schaeffer in [9]. In particular, we consider materials with hardening, for which the yield stress increases along a plastic deformation. For deformations that are not too large, the system of partial differential equations is hyperbolic. Two features of this model are: (i) shocks are not admissible in plastic regions; and (ii) a loss of hyperbolicity which is associated with the creation of shear bands. The lack of shocks in the plastic region is also observed in a model studied by Ting [13]. Trangenstein and Pember [14] studied a similar problem for one-dimensional elastoplastic solids modeled by Antman and Szymczak [1]. For this model, they observed plastic shocks during compression.

Received February 11, 1992.

1991 Mathematics Subject Classification. Primary 35L60, 35L65, 35L67, 58G16, 58G17, 65C20, 73C25, 73C 30, 73C50, 73G05. 
In this paper we describe an algorithm for the numerical resolution of elasto-plastic deformations in the context of antiplane shear models. The algorithm is tested using several examples. One of the difficulties in the numerical resolution of elasto-plastic deformations is the discontinuity in the eigenvalues and eigenvectors associated to the system. This discontinuity is introduced by the change of constitutive laws at the yield point. Our algorithm is a second-order Godunov method and is designed to solve these difficulties. We also include the description of an algorithm for the numerical resolution of the Riemann problem.

2. The model. In [9] Schaeffer discusses a model for granular flow in two space dimensions. The model is interpreted as three-dimensional antiplane shearing. The variables in this model are the $x_{3}$-component of the velocity vector, $u$, and the components of the Cauchy stress tensor, $T_{i j}, \sigma=T_{31}$ and $\tau=T_{32}$. All the other components of the stress tensor and velocity vector vanish. In the antiplane shear model the variables are functions of $t, x_{1}$, and $x_{2}$. A reduced system for deformations with dependency on only one direction, $x$, is given by the system of equations

$$
\begin{aligned}
\partial_{t} u & =c^{2} \partial_{x} \sigma, \\
\partial_{t}(\sigma+H(\gamma)) & =\partial_{x} u, \\
\partial_{t}(\tau-\alpha H(\gamma)) & =0 .
\end{aligned}
$$

Here we have approximated the yield surface by the tangent plane and made an appropriate choice of coordinates. The yield stress, $\gamma$, is considered as a history parameter and is subject to a dynamical evolution

$$
\partial_{t} \gamma= \begin{cases}\left.\partial_{t}(\sigma+\alpha \tau)\right|_{+} & \text {if } \sigma+\alpha \tau=\gamma, \\ 0 & \text { if } \sigma+\alpha \tau<\gamma,\end{cases}
$$

where $\left.d\right|_{+}=d$ if $d>0$ and $\left.d\right|_{+}=0$ if $d \leq 0$. We notice that the system (1)(2) is in conservation form even though the full two-dimensional system of [9] is not. Following the notation in [9], we say that the material is loading if $\partial_{t} \gamma>0$; otherwise, the material is unloading.

An interpretation of $H(\gamma)$ is given by comparing system (1)(2) with the full twodimensional system of Schaeffer [9]. In this case $H^{\prime}(\gamma)$ is analogous to the inverse of the hardening modulus. In what follows, $H(\gamma)$ will satisfy the convexity condition $H^{\prime \prime}(\gamma) \geq 0$ when $\gamma<\gamma_{\infty}$ for some $\gamma_{\infty} \leq+\infty$. Some examples are:

(i) $H^{\prime}(\gamma)=a^{2}$,

(ii) $H^{\prime}(\gamma)=a^{2}(b-\gamma)^{-1}$,

(iii) $H^{\prime}(\gamma)=a^{2}(b-\gamma)^{-1 / 2}$,

(iv) $H^{\prime}(\gamma)=a^{2}(\gamma-b)$,

where $a$ and $b$ are material parameters. The first three cases are applicable to granular flow [9], [10]. If $H(\gamma)$ is given by (3-iv), then system (1) with $\alpha=0$ 
reduces to a model for longitudinal deformation discussed by Lee [7]. In this setting, the variables of the system are velocity and stress in the $x$-direction.

Next we calculate the characteristic speeds of (1). For a continuous solution $(U, \gamma)$, with $U=\left(\begin{array}{c}u \\ \tau \\ \tau\end{array}\right)$, we have

$$
\left(\begin{array}{ccc}
1 & 0 & 0 \\
0 & 1+k & \alpha k \\
0 & -\alpha k & 1-\alpha^{2} k
\end{array}\right) U_{t}=\left(\begin{array}{ccc}
0 & c^{2} & 0 \\
1 & 0 & 0 \\
0 & 0 & 0
\end{array}\right) U_{x}
$$

with

$$
k(\gamma)= \begin{cases}H^{\prime}(\gamma) & \text { if loading } \\ 0 & \text { if unloading }\end{cases}
$$

and a history of parameter evolution:

$$
\gamma_{t}= \begin{cases}\left.(\sigma+\alpha \tau)_{t}\right|_{+} & \text {if loading } \\ 0 & \text { if unloading }\end{cases}
$$

or $\gamma(x, t)=\max \{\sigma(x, s)+\alpha \tau(x, s)$ for $s \leq t\}$.

If we write (4) as $A U_{t}=D U_{x}$, the eigenvalues associated to system (4) are defined as those values of $\lambda$ for which the matrix $\lambda A-D$ has zero determinant. An easy algebraic calculation shows that the eigenvalues are $\lambda=0$ and $\lambda= \pm c \sqrt{\eta}$ with

$$
\eta=\frac{1-k \alpha^{2}}{1+\left(1-\alpha^{2}\right) k}
$$

The eigenvalues are real whenever

$$
\begin{aligned}
\frac{-1}{1-\alpha^{2}}<k<\frac{1}{\alpha^{2}} & \text { if } \alpha^{2}<1, \\
k<1 & \text { if } \alpha^{2}=1, \\
k<\frac{1}{\alpha^{2}} \text { or } k>\frac{1}{\alpha^{2}-1} & \text { if } \alpha^{2}>1 .
\end{aligned}
$$

In the antiplane shear model, $\alpha$ is small. Therefore, we can safely assume that $\alpha^{2}<$ 1. In this case the system is hyperbolic if $k(\gamma)$ satisfies $-\left(\alpha^{2}-1\right)^{-1}<k(\gamma) \leq \alpha^{-2}$. As explained by Schaeffer [9] the loss of hyperbolicity in the particular case $k=$ $\alpha^{-2}$ can be associated with the development of shear bands in the material. The other case, in which $k$ decreases to $\left(\alpha^{2}-1\right)^{-1}$, would produce waves with infinite speed in general. For hardening functions that satisfy $H^{\prime \prime}(\gamma) \geq 0$, it is irrelevant since $\partial_{t} k(\gamma)=k^{\prime}(\gamma) \gamma_{t} \geq 0$ from (5).

3. Rarefactions. For continuous solutions we write system (1), while it is hyperbolic, as

$$
U_{t}+B U_{x}=0
$$




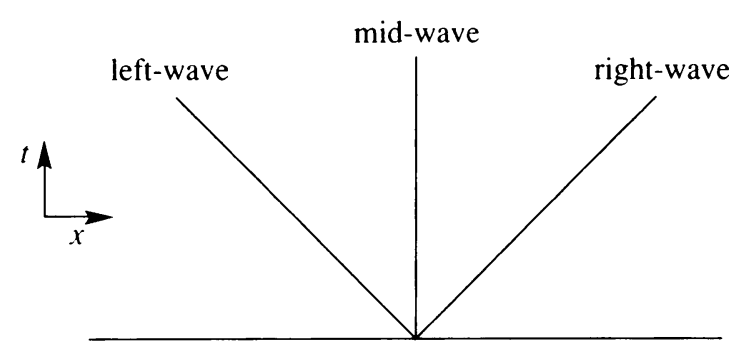

FIG. 1. Wave families

where

$$
\begin{aligned}
B & =\left(\begin{array}{ccc}
1 & 0 & 0 \\
0 & 1+k & \alpha k \\
0 & -\alpha k & 1-\alpha^{2} k
\end{array}\right)^{-1}\left(\begin{array}{ccc}
0 & -c^{2} & 0 \\
-1 & 0 & 0 \\
0 & 0 & 0
\end{array}\right) \\
& =\frac{-1}{1+\left(1-\alpha^{2}\right) k}\left(\begin{array}{ccc}
0 & c^{2}\left(1+\left(1-\alpha^{2}\right) k\right) & 0 \\
1-\alpha^{2} k & 0 & 0 \\
\alpha k & 0 & 0
\end{array}\right) .
\end{aligned}
$$

The eigenvalues of $B$ are $-c \sqrt{\eta}, 0$, and $c \sqrt{\eta}$, with corresponding right eigenvectors

$$
R_{-}=\left(\begin{array}{c}
c \sqrt{\eta} \\
\eta \\
\alpha k \\
\eta \frac{\alpha k}{1-\alpha^{2} k}
\end{array}\right), \quad R_{0}=\left(\begin{array}{l}
0 \\
0 \\
1
\end{array}\right), \quad R_{+}=\left(\begin{array}{c}
c \sqrt{\eta} \\
-\eta \\
-\eta \frac{\alpha k}{1-\alpha^{2} k}
\end{array}\right) .
$$

We refer to the waves associated to the eigenvalues $-c \sqrt{\eta}, 0$, and $c \sqrt{\eta}$ as the leftwaves, mid-waves, and right-waves respectively (see Fig. 1).

We write the gradient vector as $\nabla=(\partial / \partial u, \partial / \partial \sigma, \partial / \partial \tau)$. We say that the wave family associated to the eigenvalue-eigenvector pair $(\lambda, R)$ is genuinely nonlinear in a domain if $R \cdot \nabla \lambda \neq 0$ for all states in that domain. We say that the wave family is linearly degenerate in a domain if $R \cdot \nabla \lambda \equiv 0$ for all states in that domain (see Smoller [12]).

Proposition 1. The mid-wave family is linearly degenerate and the left- and rightwave families are genuinely nonlinear during loading whenever $H^{\prime}(\gamma)<\alpha^{-2}$ and $H^{\prime \prime}(\gamma) \neq 0$.

Proof. For the mid-wave family $\nabla \lambda=0$ and therefore this family is linearly degenerate. For the right-wave family we write

$$
\nabla \lambda=\nabla(c \sqrt{\eta})=\frac{c}{2 \sqrt{\eta}} \nabla \eta=\frac{c}{2 \sqrt{\eta}} \frac{d \eta}{d \gamma} \nabla \gamma
$$

During unloading $\nabla \lambda=0$ and the right-wave family is also linearly degenerate. During loading, $\gamma=\sigma+\alpha \tau$. Therefore $\nabla \gamma=\left(\begin{array}{l}0 \\ 1\end{array}\right)$. Moreover, from $(6), d \eta / d \gamma=$ $-k^{\prime} /\left(1+\left(1-\alpha^{2}\right) k\right)^{2}$. Thus

$$
R \cdot \nabla \lambda=\frac{c \sqrt{\eta} k^{\prime}}{2\left(1-\alpha^{2} k\right)\left(1+\left(1-\alpha^{2}\right) k\right)^{2}},
$$


where $k^{\prime}(\gamma)=H^{\prime \prime}(\gamma)$ during loading and $k^{\prime}(\gamma)=0$ during unloading. Relation (10) holds also for left-waves. The assumptions on $H(\gamma)$ imply that $R \cdot \nabla \lambda \neq 0$ and therefore the left- and right-wave families are genuinely nonlinear.

Let $(\lambda, R)$ be an eigenvalue-eigenvector pair associated to a genuinely nonlinear wave family. For this family, a nonlinear centered rarefaction wave is a oneparameter family of states $U(\xi), \xi=\left(x-x_{0}\right) /\left(t-t_{0}\right)$, described by $d u / d \xi=\widehat{R}$, where $\widehat{R}$ is a normalization of $R$ such that $\widehat{R} \cdot \nabla \lambda=1$. For nonlinear centered rarefaction waves, we can write the eigenvalues as functions of $\xi$ by $\lambda=\lambda(U(\xi))$. We differentiate with respect to $\xi$ to obtain $d \lambda / d \xi=\nabla \lambda \cdot d U / d \xi=1$. Therefore $\lambda(\xi)=\xi$ and $|\lambda|=c \sqrt{\eta}$ must decrease in the direction of increasing time.

Proposition 2. Let $H(\gamma)$ satisfy $H^{\prime \prime}(\gamma)>0$ for $\gamma<\gamma_{\infty}$. Then $\sigma$ increases along a nonlinear centered rarefaction wave in forward time; that is, $\sigma_{t}>0$.

Proof. From (9) we see that $d \sigma / d \xi=\eta /(R \cdot \nabla \lambda)>0$. Therefore we may choose $\sigma$ as the wave parameter. We write $\frac{d \eta}{d \sigma}=\frac{d \eta}{d \gamma} \frac{d \gamma}{d \sigma}$. Now, during loading $\gamma=\sigma+\alpha \tau$ and therefore $d \gamma / d \sigma=1+\alpha d \tau / d \sigma$. From (9), along a rarefaction, we have $d \tau / d \sigma=$ $\alpha k /\left(1-\alpha^{2} k\right)$ and thus $d \gamma / d \sigma=1+\alpha d \tau / d \sigma=1 /\left(1-\alpha^{2} k\right)$. During unloading $d \gamma / d \sigma=0$.

Since $\eta$ is given in terms of $k(\gamma)$, differentiation with respect to $\gamma$ gives $d \eta / d \gamma=$ $-k^{\prime} /\left(\left(1+\left(1-\alpha^{2}\right) k\right)^{2}\right)$. Therefore, $d \eta / d \sigma=-k^{\prime} /\left(\left(1+\left(1-\alpha^{2}\right) k\right)^{2}\left(1-\alpha^{2} k\right)\right)<0$. Along a nonlinear rarefaction wave, $|\lambda|=c \sqrt{\eta}$ must decrease in the direction of increasing time. Therefore, from the previous inequality, we see that $\sigma$ is increasing in the direction of increasing time, along a nonlinear rarefaction wave.

4. Shocks. Let $\lambda$ be an eigenvalue associated to a genuinely nonlinear wave family. We define a nonlinear shock as a jump discontinuity in $(U, \gamma)$ that satisfies the Lax entropy condition for admissibility. This condition states that for $s$ the speed of the shock and for $\left(\left(U_{L}, \gamma_{L}\right)\right.$ and $\left.\left(U_{R}, \gamma_{R}\right)\right)$ the states on the left and on the right of the shock, the double inequality

must be satisfied.

$$
\lambda\left(U_{L}, \gamma_{L}\right)<s<\lambda\left(U_{R}, \gamma_{R}\right)
$$

We will show that nonlinear shocks (during plastic deformations) do not exist. For a shock with speed $s \neq 0$, we write $\left(U_{a}, \gamma_{a}\right)$ as the state after the shock and $\left(U_{b}, \gamma_{b}\right)$ as the state before the shock, in the direction of increasing time.

Theorem 3. We assume that $H^{\prime \prime}(\gamma) \geq 0$ and $H^{\prime}(\gamma) \geq 0$. Then there are no nonlinear shocks such that $\left(U_{a}, \gamma_{a}\right)$ is a plastic state.

Proof. If $\left(U_{a}, \gamma_{a}\right)$ is a plastic state, we have $\gamma_{a} \geq \gamma_{b}$. In this case, since $d \eta / d \gamma=$ $-k^{\prime} /\left(\left(1+\left(1-\alpha^{2}\right) k\right)^{2}\right)$, we have

$$
\left|\lambda\left(U_{a}, \gamma_{a}\right)\right| \leq\left|\lambda\left(U_{b}, \gamma_{b}\right)\right| .
$$

Therefore any nonlinear shock would violate the Lax entropy condition for shock admissibility.

Theorem 3 shows that during loading there are no shock waves. During unloading the material is linear and all the wave families are contact discontinuities of speed $s=\lambda\left(U_{L}, \gamma_{L}\right)=\lambda\left(U_{R}, \gamma_{R}\right)$. 
For the remainder of this paper we will consider a function $H(\gamma)$ which is monotone nondecreasing $\left(H^{\prime} \geq 0\right)$ and convex $\left(H^{\prime \prime} \geq 0\right)$. If we were to allow $H^{\prime}(\gamma)<0$ for some values of $\gamma$, we would introduce plastic waves that travel at speeds faster than the elastic waves. In this case, elastic-to-plastic shocks would exist as well as multiple solutions associated to this type of shock wave. We are only interested in deformations where the plastic waves are slower than the elastic waves.

5. Wave curves. The mid-wave is a contact discontinuity. It is described by the relations $[u]=[\sigma]=0$ with $[\tau]$ and $[\gamma]$ as free parameters.

We continue with a description on the left- and right-waves. With respect to time, we denote by $\left(U_{b}, \gamma_{b}\right)$ the state before a wave has passed and by $\left(U_{a}, \gamma_{a}\right)$ the state after the wave has passed. As shown in the previous sections, when $\sigma_{a} \leq \sigma_{b}$, the waves are linear and we have the following relations for the wave speed $s$ and the states at both sides of the wave,

$$
\begin{aligned}
& {[u]=c[\sigma], \quad[\tau]=[\gamma]=0, \quad s=-c, \quad \text { for left waves and }} \\
& {[u]=-c[\sigma], \quad[\tau]=[\gamma]=0, \quad s=c, \quad \text { for right waves. }}
\end{aligned}
$$

For $\sigma_{a}>\sigma_{b}$, we parameterize the rarefaction curves by $\sigma$. Thus the states $(U, \gamma)$ on the rarefaction will satisfy the differential equations

$$
\begin{aligned}
& \frac{d u}{d \sigma}=(+/-) \frac{c}{\sqrt{\eta}} \text { left } / \text { right } \\
& \frac{d \tau}{d \sigma}=\frac{\alpha k}{1-\alpha^{2} k} \\
& \frac{d \gamma}{d \sigma}= \begin{cases}\frac{1}{1-\alpha^{2} k} & \text { during loading } \\
0 & \text { during unloading. }\end{cases}
\end{aligned}
$$

In general these curves fail to be continuously differentiable, $C^{1}$, at the point of transition from elastic to plastic deformation. We should observe that (12) also applies to (11) where $\sigma_{a}<\sigma_{b}$ and $\eta=1$. Therefore (12) describes the complete wave curves. We further notice that integrating the last two equations in (12), along plastic loading, we obtain

$$
[\gamma]-\alpha^{2}[H(\gamma)]=[\sigma] \text { and }[\tau]=\alpha[H(\gamma)] .
$$

Thus we can write the wave curves as

$$
\begin{aligned}
& {[u]=(+/-) \int_{\sigma_{0}}^{\sigma} \frac{c}{\sqrt{\eta(\gamma(\psi))}} d \psi \quad \text { left/right }} \\
& {[\tau]= \begin{cases}\alpha[H(\gamma)] & \text { during loading } \\
0 & \text { during unloading. }\end{cases} } \\
& {[\gamma]= \begin{cases}{[\sigma]+\alpha^{2}[H(\gamma)]} & \text { during loading } \\
0 & \text { during unloading. }\end{cases} }
\end{aligned}
$$

6. Riemann solver. A Riemann problem is the initial value problem defined by a state in which the material on the left of the origin, $x<0$, is in a constant state, $U_{L}$, and likewise for $x>0$, the material is in a constant state, $U_{R}$. In the next 
section we will describe an algorithm for the resolution of the hyperbolic system (1). This algorithm is of Godunov type. The resolution of Riemann problems is a fundamental step in algorithms of this type $[15,6]$ as well as other methods (e.g., the random choice method $[3,5,8]$, front tracking $[2,5], \ldots)$.

In this section we will describe an algorithm for the numerical resolution of the Riemann problem. In general, the numerical algorithms for the solution of hyperbolic systems can also make use of approximate solutions to Riemann problems. Following this reason, we will also describe a linearized version of this Riemann solver.

The lack of $C^{1}$ continuity on the wave curves at the yield point is also seen as a jump on the slopes, across a rarefaction wave, from an elastic to a plastic state, in forward time. Let $\left(U_{b}, \gamma_{b}\right)$ be an elastic state and $\left(U_{a}, \gamma_{a}\right)$ a plastic state. We parameterize the rarefaction wave as $(U(s), \gamma(s)), s_{b} \leq s \leq s_{a}$. If for $s_{0}, s_{b}<s_{0}<$ $s_{a},\left(U\left(s_{0}\right), \gamma\left(s_{0}\right)\right)$ is the first state in time on the yield surface, we have

$$
\lim _{s \rightarrow s_{0}^{-}} \lambda^{2}(U(s), \gamma(s))=c^{2} \neq \lim _{s \rightarrow s_{0}^{+}} \lambda^{2}(U(s), \gamma(s)) .
$$

In the $(x, t)$-plane the rarefaction fan consists of an elastic linear wave, followed by a plastic (nonlinear) rarefaction and a constant state $\left(U\left(s_{0}\right), \gamma\left(s_{0}\right)\right)$ between both waves (see Fig. 2).

In this situation we can think of the right- and left-waves, each as a group of two waves, one elastic and the other plastic. In the case of a purely elastic or plastic deformation one of these two waves would be trivial, having zero strength.

Given the initial left and right states $\left(U_{L}^{0}, \gamma_{L}^{0}\right)$ and $\left(U_{R}^{0}, \gamma_{R}^{0}\right)$, the solution of the Riemann problem consists of two groups of waves (left and right) each consisting of a fast elastic wave and a slow plastic wave with three constant states. The final states $U_{L, R}^{2}$ correspond to two different material points with possibly different history $(\gamma$ and $\tau$ ) and identical velocity and directional stress $\sigma$ (see Fig. 3 on p. 296).

We define the functional

$$
F\left(\sigma ; U_{L}^{0}, \gamma_{L}^{0} ; U_{R}^{0}, \gamma_{R}^{0}\right)=u_{L}^{2}\left(\sigma ; U_{L}^{0}, \gamma_{L}^{0}\right)-u_{R}^{2}\left(\sigma ; U_{R}^{0}, \gamma_{R}^{0}\right)
$$

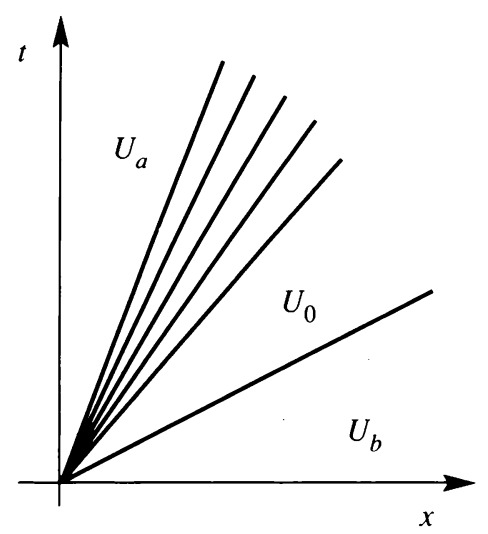

FIG. 2. Elastic-plastic rarefaction 


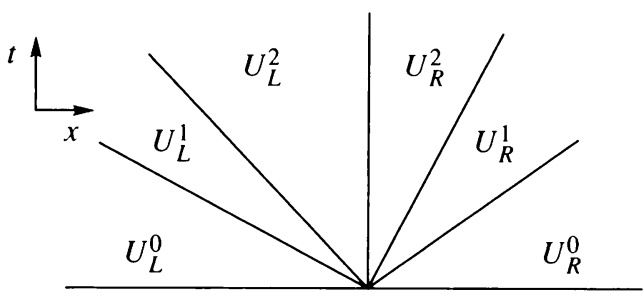

FIG. 3. Riemann problem

where

$$
u_{L}^{2}=u_{L}^{0}+\varphi\left(\sigma ; U_{L}^{0}, \gamma_{L}^{0}\right), \quad u_{R}^{2}=u_{R}^{0}-\varphi\left(\sigma ; U_{R}^{0}, \gamma_{R}^{0}\right)
$$

and

$$
\varphi\left(\sigma ; U_{R}^{0}, \gamma_{R}^{0}\right)=c \int_{\sigma_{0}}^{\sigma} \frac{d s}{\sqrt{\eta(\gamma(s))}} .
$$

where $\gamma=\gamma(\sigma)$ is given implicitly by the third equation of

$$
\begin{aligned}
& \frac{d \varphi}{d \sigma}=\frac{c}{\sqrt{\eta(\gamma)}}, \\
& \frac{d \tau}{d \sigma}=\frac{\alpha k(\gamma)}{1-\alpha^{2} k(\gamma)}, \\
& \frac{d \gamma}{d \sigma}=\frac{1}{1-\alpha^{2} k(\gamma)} .
\end{aligned}
$$

From the definition of $\eta(5)(6)$ we observe that the right-hand side of (16) is discontinuous at the point of transition from elastic to plastic deformation. An important consequence of this fact is that any attempt to linearize (16) at states near yield could fail since the right-hand side of (16) changes abruptly. This is of particular interest when designing numerical algorithms for the resolution of system $(1)(2)$.

A solution of the Riemann problem corresponds to a root of

$$
F\left(\sigma ; U_{L}^{0}, \gamma_{L}^{0} ; U_{R}^{0}, \gamma_{R}^{0}\right)=0 \text {. }
$$

It is worthwhile noticing that $\frac{d F}{d \sigma}=\frac{d \varphi}{d \sigma}\left(\sigma ; U_{L}^{0}, \gamma_{L}^{0}\right)+\frac{d \varphi}{d \sigma}\left(\sigma ; U_{R}^{0}, \gamma_{R}^{0}\right)>0$ and therefore, if there is a solution of (17), it will be unique.

For $\sigma^{*}$ such that $F\left(\sigma^{*} ; U_{L}^{0}, \gamma_{L}^{0}, U_{R}^{0}, \gamma_{R}^{0}\right)=0$ the solution of the Riemann problem is given by

$$
\begin{aligned}
\sigma_{L}^{2} & =\sigma^{*} & \sigma_{R}^{2} & =\sigma^{*} \\
u_{L}^{2} & =u_{L}^{0}+\varphi\left(\sigma^{*} ; U_{L}^{0}, \gamma_{L}^{0}\right) & u_{R}^{2} & =u_{R}^{0}-\varphi\left(\sigma^{*} ; U_{R}^{0}, \gamma_{R}^{0}\right) \\
\gamma_{L}^{1} & =\gamma_{L}^{0} & \gamma_{R}^{1} & =\gamma_{R}^{0} \\
\tau_{L}^{1} & =\tau_{L}^{0} & \tau_{R}^{1} & =\tau_{R}^{0}
\end{aligned}
$$

and the other components are found during the integration of (16).

For certain initial states the Riemann problem fails to have a solution. To explain this, consider initial left and right states that are close to the yield surface. If the wave curves from the left and right states approach each other in the direction of 
increasing stress, we may reach the shear point before the wave curves intersect. This is seen from integrating (16) along a path of plastic loading.

If we define $\Phi(\gamma)$ by $\Phi(\gamma(\sigma))=\varphi(\sigma)$, we see that

$$
d \Phi=\left(1+\left(1-\alpha^{2}\right) H^{\prime}(\gamma)\right)^{1 / 2}\left(1-\alpha^{2} H^{\prime}(\gamma)\right)^{1 / 2} d \gamma .
$$

In the appendix we show how the shear point is reached for finite values of $\sigma$ for different types of $H(\gamma)$. In order to illustrate the nonexistence of solutions of the Riemann problem for some initial data, we now consider a particular case. We assume that $\alpha \neq 0$ and there is a finite value $\gamma_{\mathrm{P}}$ of the yield stress at which the material fails due to shear, that is $H^{\prime}\left(\gamma_{\mathrm{P}}\right)=\alpha^{-2}$ (see the appendix for a more detailed description). From (13) we can write

$$
\gamma-\gamma^{0}=\sigma-\sigma_{\max }^{0}+\alpha^{2}\left(H(\gamma)-H\left(\gamma_{0}\right)\right)
$$

where $\left(U^{0}=\left(\begin{array}{c}u^{0} \\ \sigma^{0} \\ \tau^{0}\end{array}\right), \gamma^{0}\right)$ is the initial state and $\sigma_{\max }^{0}=\gamma^{0}+\alpha \tau^{0}$ is the maximum value of $\sigma$ for which a state on the wave curve through $\left(U^{0}, \gamma^{0}\right)$ is in the elastic regime. In (19) we solve for $\sigma$ and we find $\sigma_{\mathrm{P}}$, the maximum allowed stress before shear failure,

$$
\sigma_{\mathrm{P}}=\gamma_{\mathrm{P}}-\alpha^{2}\left(H\left(\gamma_{\mathrm{P}}\right)-H\left(\gamma_{0}\right)\right)-\alpha \tau_{0} .
$$

Integrating (18) during loading we obtain

$$
\varphi(\sigma)=\varphi\left(\sigma_{0}\right)+\int_{\gamma_{0}}^{\gamma(\sigma)}\left(1+\left(1-\alpha^{2}\right) H^{\prime}(\gamma)\right)^{1 / 2}\left(1-\alpha^{2} H^{\prime}(\gamma)\right)^{1 / 2} d \gamma,
$$

which defines $\varphi(\sigma)$ for $\sigma \leq \sigma_{\mathrm{P}}$. Furthermore $\varphi\left(\sigma_{\mathrm{P}}\right)$ is bounded and $\lim _{\sigma \rightarrow \sigma_{\mathrm{p}}} \varphi^{\prime}(\sigma)=$ $+\infty$. Under these circumstances it is possible that the wave curves through the left and right states do not intersect and the corresponding Riemann problem has no solution. In Fig. 4 we show the projection on the $(\sigma, u)$-plane of wave curves through initial states $\left(U_{L}, \gamma_{L}\right)$ and $\left(U_{R}, \gamma_{R}\right)$ that do not intersect in the hyperbolic region.

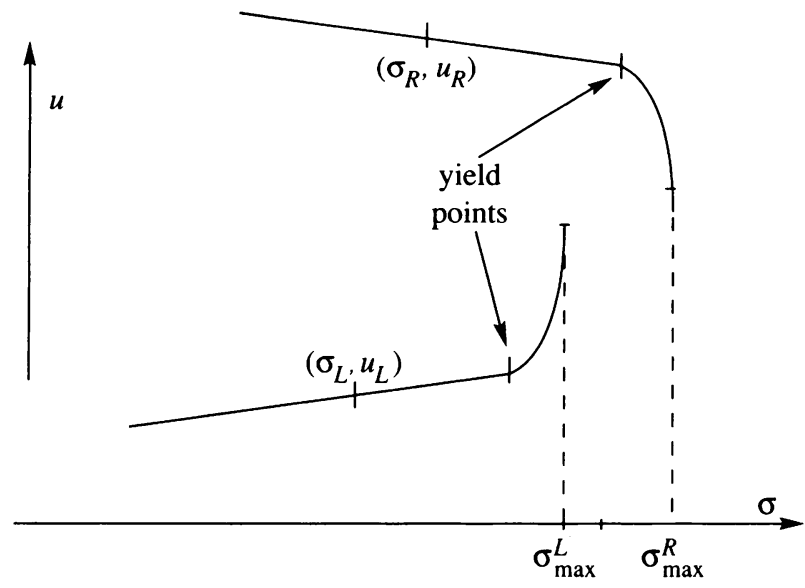

FIG. 4. Riemann problem with no solution 


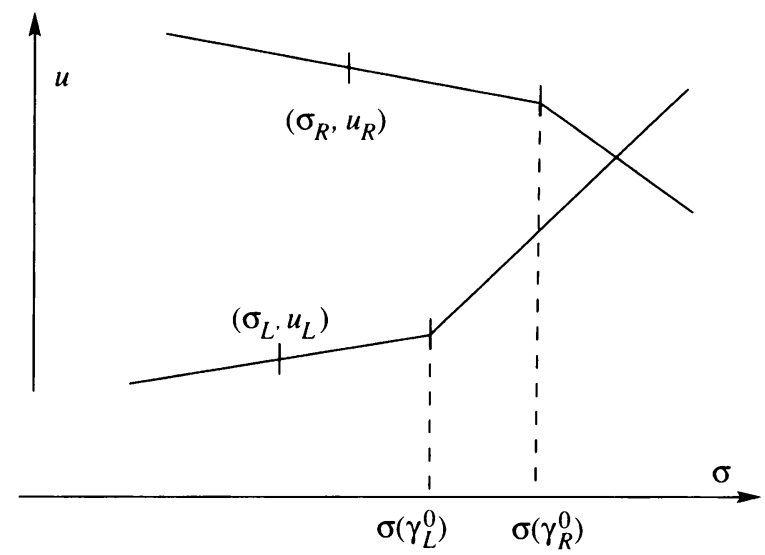

FIG. 5. Approximate Riemann problem

As was mentioned earlier, the loss of hyperbolicity in system (1) is associated with the formation of shear bands in the material. In this article we consider only the case when the system is hyperbolic. It suffices to mention that for data for which the solution fails to exist, it is possible to nonetheless solve the problem by introducing a shear band, whose evolution is governed by separate equations. For a model of the shear band evolution proposed by Schaeffer [9], some preliminary analytical [11] and numerical [4] work has been performed.

An approximate Riemann solver. In some circumstances, only an approximation to the solution of a Riemann problem is needed. We now describe a Linearized Riemann Solver. As has been shown in the previous section, any linearization of the Riemann problem may offer solutions for specific left and right states for which the material actually moves into failure. Nevertheless, away from the shear points, and for initial states that are close enough, the approximation is still valuable for certain problems.

The nonlinearity of the system in loading is given by the hardening-related function $H(\gamma)$. Given left and right states $\left(U_{L}^{0}, \gamma_{L}^{0}\right)$ and $\left(U_{R}^{0}, \gamma_{R}^{0}\right)$, we simplify the Riemann problem by linearizing $H(\gamma)$. We think of the left and right states as two material points with different histories. Then we linearize $H(\gamma)$, on each side, about $\gamma_{L}^{0}$ and $\gamma_{R}^{0}$ respectively. The wave curves given by (13) or (16) are piecewise linear (with two components, see Fig. 5), but with different slopes. In particular for elastic states, $k(\gamma)=0$, and for plastic states $k(\gamma)=k\left(\gamma_{L}^{0}\right)$ on the left side and $k(\gamma)=k\left(\gamma_{R}^{0}\right)$ on the right side.

The function $F\left(\sigma ; U_{L}^{0}, \gamma_{L}^{0} ; U_{R}^{0}, \gamma_{R}^{0}\right)$ as defined in (14) is affine on $\sigma$. The root $\sigma^{*}$ of (17) always exists and can be solved explicitly. The rest of the algorithm follows as in the nonlinear case.

7. Hyperbolic solver. In this section we describe a numerical algorithm to compute solutions to system (1-2). We will follow a formulation in the spirit of Yee [18, 17] of a method that is of MUSCL type and is second-order accurate on time and space. 


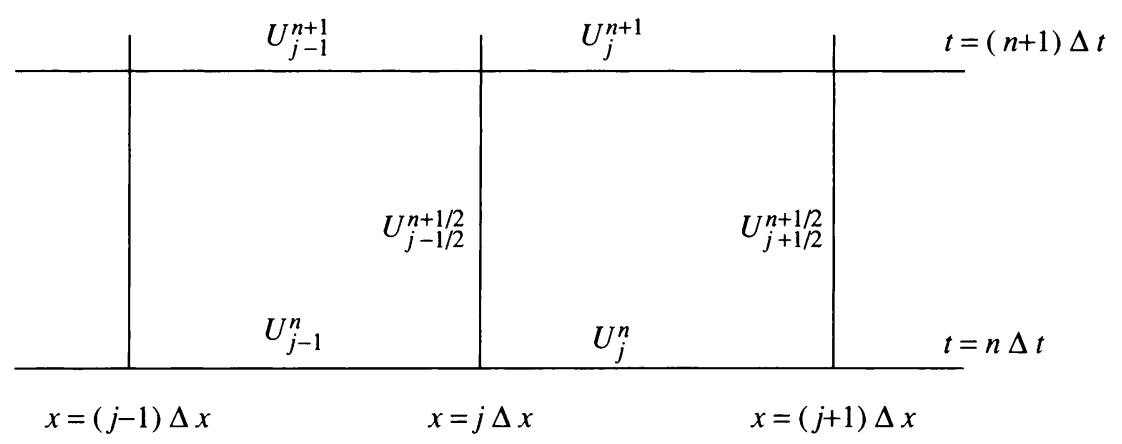

FIG. 6. Grid cells

To describe the algorithm, first we write system (1) as

$$
G(U)_{t}+F(U)_{x}=0
$$

with $G(U)=\left(\begin{array}{c}u \\ \sigma+H(\gamma) \\ \tau-\alpha H(\gamma)\end{array}\right)$ and $F(U)=\left(\begin{array}{c}-c^{2} \sigma \\ -u \\ 0\end{array}\right)$.

We write $U_{j}^{n}$ as the numerical approximation to the average of the solution to system (1) in the interval $(j \Delta x,(j+1) \Delta x)$ at time $t=n \Delta t$ (see Fig. 6).

We assume $\left\{U_{j}^{n}\right\}_{j}$ to be known for all values of $j$, and we want to evaluate $\left\{U_{j}^{n+1}\right\}_{j}$. We denote by $U_{j+\frac{1}{2}}^{n+\frac{1}{2}}$ the constant state at the cell edge given by $x=(j+1) \Delta x$. At this point we will assume these states to be known for the sake of the following argument. The rest of this section will be devoted to obtaining such states.

Integrating the conservation law (21) on the $j$ th cell we obtain an algorithm for the calculation of $U_{j}^{n+1}$ :

$$
G\left(U_{j}^{n+1}\right)=G\left(U_{j}^{n}\right)-\frac{\Delta t}{\Delta x}\left(F\left(U_{j+\frac{1}{2}}^{n+\frac{1}{2}}\right)-F\left(U_{j-\frac{1}{2}}^{n+\frac{1}{2}}\right)\right)=G\left(U_{j}^{n}\right)+F_{j}^{n} .
$$

At this point two issues remain to be solved: (i) how to obtain the states $U_{j+\frac{1}{2}}^{n+\frac{1}{2}}$ at the cell edges; and (ii) how to obtain $U_{j}^{n+1}$ from (22) once $\left\{U_{j+\frac{1}{2}}^{n+\frac{1}{2}}\right\}_{j}$ are known. We start with (b) assuming that we know $\left\{U_{j+\frac{1}{2}}^{n+\frac{1}{2}}\right\}_{j}$. We notice that $G(U)=U$ only while $U$ is an elastic state. A first idea would be to work with the conserved quantities $(\sigma+H(\gamma), \tau-\alpha H(\gamma))$ instead of stresses. However, this selection of variables brings some oscillations in the numerical calculations due to the fact that the history parameter is given in terms of the stresses and we will need to invert the conserved quantities into stresses. We choose to work with stresses and provide an easy algorithm for the updating. Given a state $\left(U^{0}, \gamma^{0}\right)$, we calculate the maximum stress allowed for an elastic state, $\sigma_{\max }^{0}=\gamma^{0}-\alpha \tau^{0}$. The second and third equations in (22) are of the type:

$$
\begin{aligned}
\sigma^{1}+H\left(\gamma^{1}\right) & =\sigma^{0}+H\left(\gamma^{0}\right)+f_{\sigma}, \\
\tau^{1}-\alpha H\left(\gamma^{1}\right) & =\tau^{0}-\alpha H\left(\gamma^{0}\right) .
\end{aligned}
$$


If $\sigma^{0}+f_{\sigma} \leq \sigma_{\max }^{0}$, the deformation is still elastic and we can write

$$
\sigma^{1}=\sigma^{0}+f_{\sigma}, \quad \tau^{1}=\tau^{0}, \quad \gamma^{1}=\gamma^{0} .
$$

Otherwise we split $f_{\sigma}$ into an elastic $f_{\mathrm{e}}=\sigma_{\max }^{0}-\sigma^{0}$ and a plastic flux $f_{\mathrm{p}}=f_{\sigma}-f_{\mathrm{e}}$. The elastic flux is absorbed to reach the yield point by an elastic deformation. Then a plastic deformation follows:

$$
\begin{aligned}
\sigma^{1}+H\left(\gamma^{1}\right) & =\sigma_{\max }^{0}+H\left(\gamma^{0}\right)+f_{\mathrm{p}}, \\
\tau^{1}-\alpha H\left(\gamma^{1}\right) & =\tau^{0}-\alpha H\left(\gamma^{0}\right), \\
\gamma^{1} & =\sigma^{1}+\alpha \tau^{1} .
\end{aligned}
$$

Thus we need to solve the implicit equation in $\gamma^{1}$ :

$$
\Sigma\left(\gamma^{1}\right) \equiv \gamma^{1}+\left(1-\alpha^{2}\right) H\left(\gamma^{1}\right)=\gamma^{0}+\left(1-\alpha^{2}\right) H\left(\gamma^{0}\right)+f_{\mathrm{p}} .
$$

Since $\alpha^{2}<1$ and $H^{\prime}(\gamma) \geq 0$, we observe that $d \Sigma / d \gamma=1+\left(1-\alpha^{2}\right) H^{\prime}(\gamma)>0$. The monotonicity of $\Sigma(\gamma)$ makes the numerical resolution of (23) trivial. Once $\gamma^{1}$ is known, we obtain $\sigma^{1}$ and $\tau^{1}$ from (23).

We now give a description of how to obtain $U_{j+\frac{1}{2}}^{n+\frac{1}{2}}$. We first find the states on the $j$ th cell at an intermediate time by an iteration of Godunov type:

$$
G\left(U_{j}^{n+\frac{1}{2}}\right)=G\left(U_{j}^{n}\right)+\frac{\Delta t}{2 \Delta x}\left(F\left(U R_{j}^{*}\right)-F\left(U L_{j}^{*}\right)\right)
$$

where $U L_{j}^{*}$ and $U R_{j}^{*}$ are chosen to satisfy

$$
G\left(U L_{j}^{*}\right)=G\left(U_{j}^{n}\right)-g_{j} / 2 \text { and } G\left(U R_{j}^{*}\right)=G\left(U_{j}^{n}\right)+g_{j} / 2,
$$

and $g_{j}$ is defined by

$$
g_{j}=\operatorname{ave}\left(U_{j+1}^{n}-U_{j}^{n}, U_{j}^{n}-U_{j-1}^{n}\right) .
$$

The ave function, introduced by Van Leer [16] is defined as

$$
\text { ave }(a, b)= \begin{cases}0 & \text { if } a b \leq 0 \text { or } a(a-b) \leq 0 \\ 2 \operatorname{sign}(a) \min \{|a|,|b|,|a-b|\} & \text { otherwise }\end{cases}
$$

We use $U_{j}^{n+\frac{1}{2}}$ as the first step toward building a Godunov integration (22) where the flux is conserved. We determine the state $U_{j-\frac{1}{2}}^{n+\frac{1}{2}}$ as the (exact or linearized) solution on the ray $x=j \Delta x$ of the Riemann problem with initial states $\left(U L_{j}, U R_{j}\right)$ defined implicitly by

$$
G\left(U L_{j}\right)=G\left(U_{j-1}^{n+\frac{1}{2}}\right)+\frac{g_{j-1}}{2} \text { and } G\left(U R_{j}\right)=G\left(U_{j}^{n+\frac{1}{2}}\right)-\frac{g_{j}}{2} .
$$

8. Numerical calculations. In the following sections we will show several examples of numerical experiments on test problems.

8.1. Scale-invariant problems. Scale-invariant problems studied by Schaeffer and Shearer [10] consist of system (1) with $\alpha=0$ and initial data that is continuous and piecewise linear. Schaeffer and Shearer construct piecewise linear solutions of this 
problem. As in Lee [7], the speed $s$ of a transition front from an elastic state to a plastic state is given by

$$
s=\frac{\partial_{x} v_{\mathrm{E}}}{\partial_{x} \sigma_{\mathrm{E}}-\partial_{x} \gamma_{\mathrm{E}}},
$$

where the $\left(v_{\mathrm{E}}, \sigma_{\mathrm{E}}, \gamma_{\mathrm{E}}\right)$ is the elastic state. We compute the numerical experiment with the following parameters:

- computational domain $-1<x<1$ and $t>0$,

- parameters: $\alpha=0, c=100$, and $H(\gamma)=\gamma / 0.27$,

- initial data: $v(x, 0)=90 x, \sigma(x, 0)=-1.1|x|, \gamma(x, 0)=-|x|$,

- since the initial data and the expected solution are piecewise linear, we extrapolate linearly the values of the solution in the interior of the computational domain to the boundary.

In Fig. 7 (see p. 302) we show the initial states and the states after 10 and 210 time steps. The sound speed in these calculations is 100 . We observe two transition waves; first an elastic-to-plastic (loading) front that travels at a speed of $\approx 901.2$ and a plastic-to-elastic (unloading) front that travels at a speed of $\approx 71.23$. Both speeds are in the range predicted by the analysis of this problem, 900 and 71.878 respectively. In Fig. 8 (see p. 303) we do a comparison with the exact solution given by Schaeffer and Shearer analysis.

8.2. Quasi-static solutions. A second numerical experiment is performed for a solution to system (1) with initial values that are constant for $\sigma, \tau$, and $\gamma$, and linear for the velocity. For such initial values the solution will satisfy $v_{t}=0, \sigma_{x}=$ $0, \tau_{x}=0$, and $\gamma_{x}=0$ for all $(x, t)$. Since the solution is expected to be linear in $x$ we choose conditions on the boundary that are a linear extrapolation of the values in the interior of the domain.

We perform the numerical experiment with the following parameters:

- computational domain $-1<x<1$ and $t>0$,

- parameters: $\alpha=0.3, c=100$, and $H^{\prime}(\gamma)=2 /(3 \sqrt{1-\gamma})$,

- initial data: $v(x, 0)=10 x, \sigma(x, 0)=0.265, \gamma(x, 0)=0.3$, and $\tau(x, 0)=$ 0.1 .

At $t=0.0005$, the deformation becomes plastic. The evolution of the solution is shown in Fig. 9 (see p. 304). 

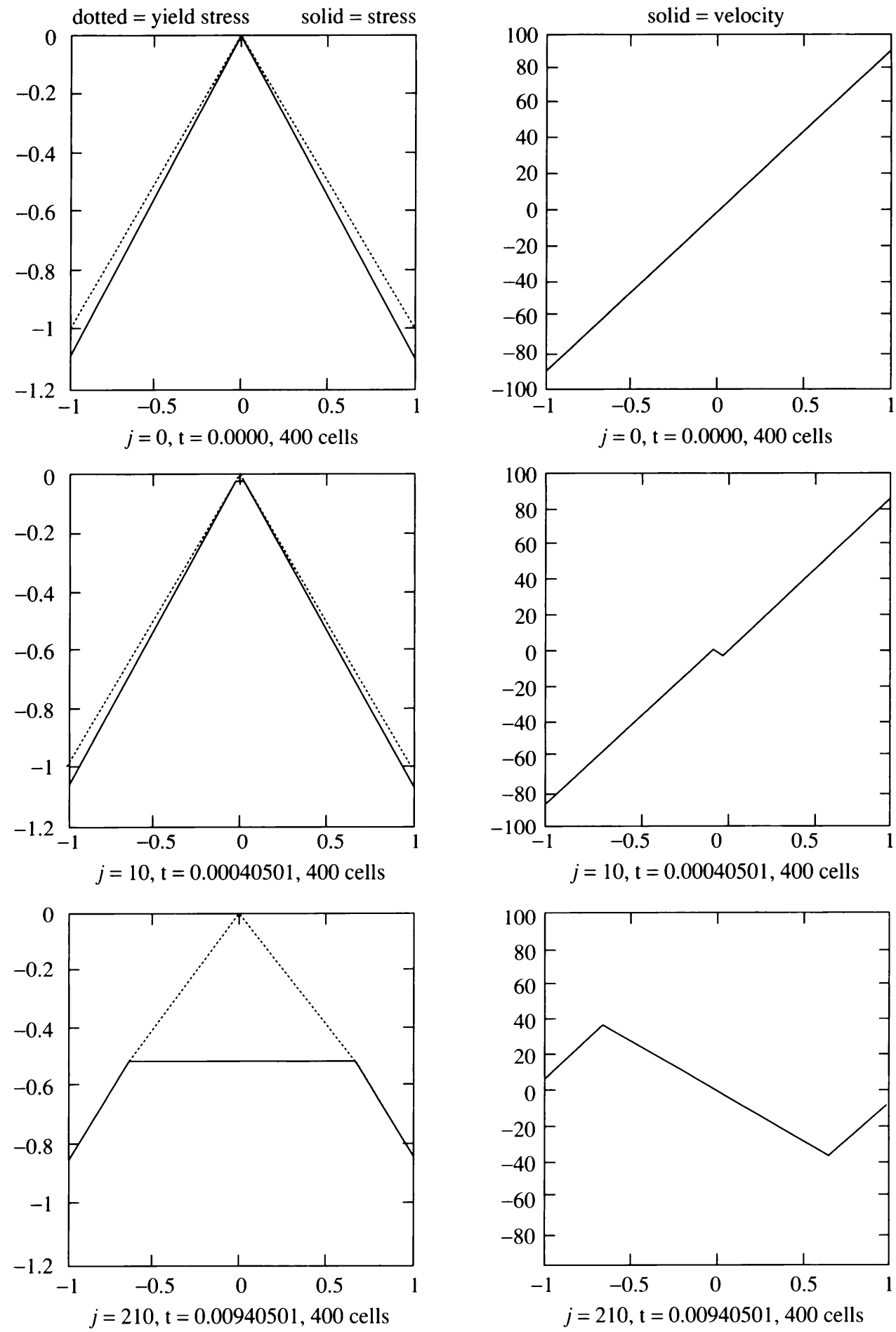

FIG. 7. Scale-invariant deformations 

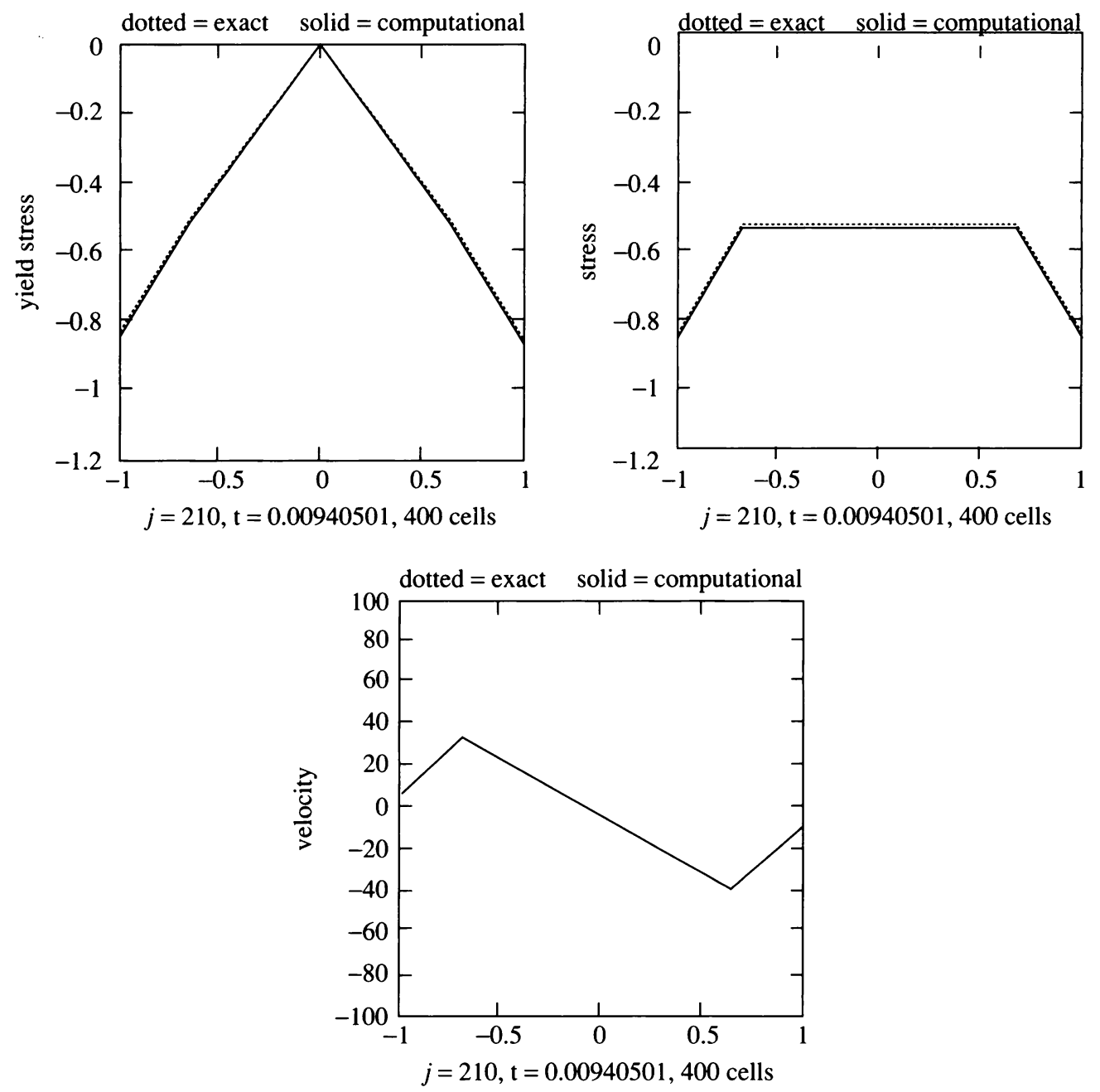

Fig. 8. Scale-invariant deformation comparison 

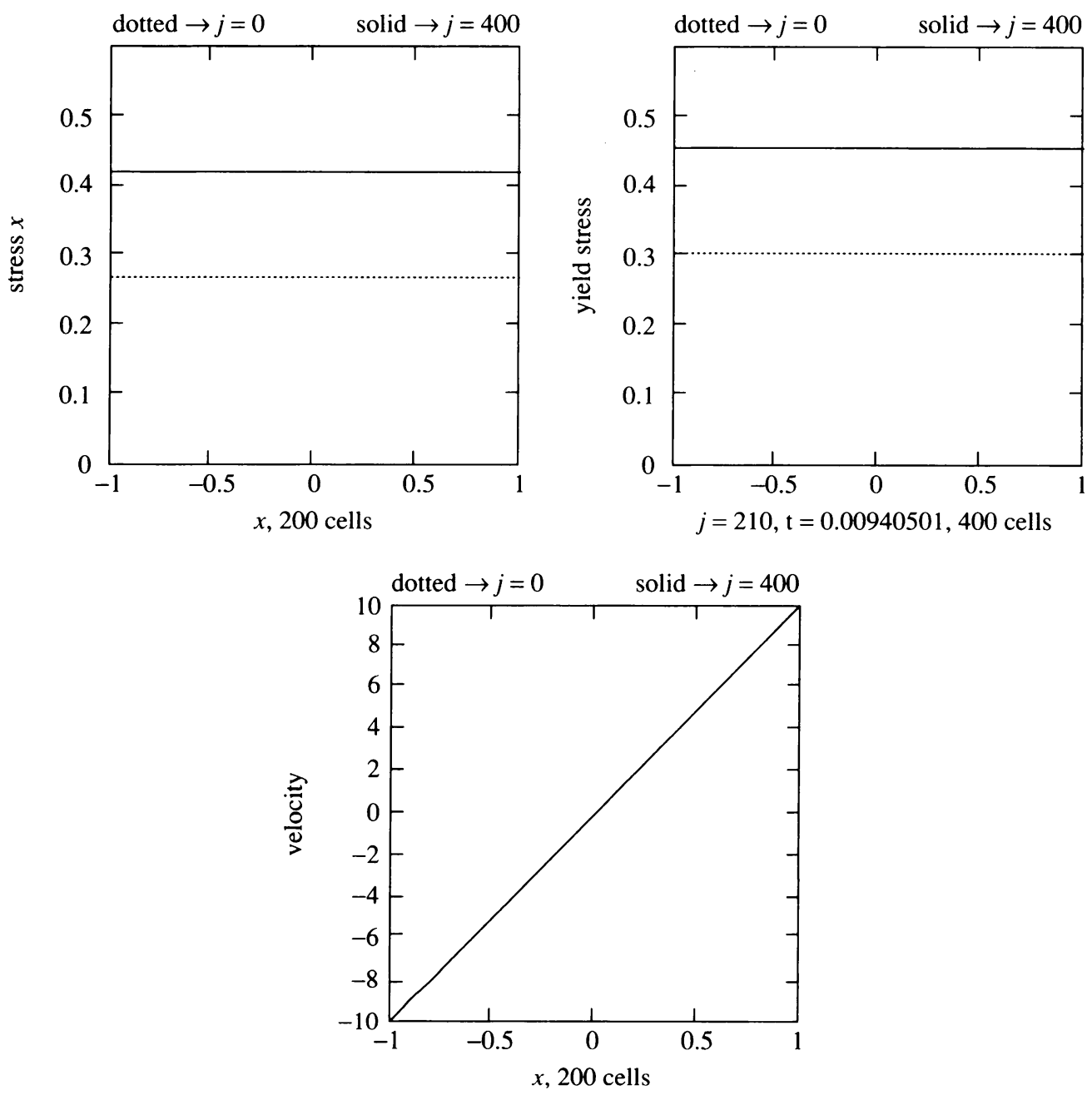

FIG. 9. Quasi-static deformations

8.3. Lee's problem for a loading rod under impact. In [7], Lee studied the problem of propagation of plastic waves in a rod, resulting from the normal impact at an end of the rod. The hyperbolic system describing such deformations formally resembles system (1):

$$
\begin{aligned}
& v_{t}=-\rho^{-1} \sigma_{x}, \\
& \varepsilon_{t}=-v_{x},
\end{aligned}
$$

where $v$ is the velocity in the $x$ direction, $\varepsilon$ is the strain, $\sigma$ is the stress, and $\rho$ is the density. The stress-strain relation $\left(\sigma=\sigma_{\mathrm{p}}(\varepsilon)\right)$ is given by a typical curve (see [7]) as in Fig. 10. The slope of the linear segment $O A$ is $E$, the Young Modulus, and $Y$ is the initial yield stress. This curve has a continuous derivative at the yield 
stress $Y$. The stress-strain relation is described by

$$
\sigma(x, t)=\sigma(\varepsilon(x, t))= \begin{cases}\sigma_{\mathrm{p}}(\varepsilon) & \text { if } \varepsilon=\varepsilon_{\max } \\ \sigma_{\mathrm{p}}\left(\varepsilon_{\max }\right)-E\left(\varepsilon_{\max }-\varepsilon\right) & \text { if } \varepsilon<\varepsilon_{\max }\end{cases}
$$

where $\varepsilon_{\max }=\max \{\varepsilon(x, s)$ for $s \leq t\}$.

The impact problem is modeled by prescribing zero initial conditions on the stress and velocity and boundary conditions which are a given constant velocity on the left boundary and zero stress on the right boundary. Since no analytic expression is given in [7] for the stress-strain relation we rescale the problem and look for solutions that are qualitatively similar to the solution obtained from Lee's analysis. Thus we rescale the problem by $c_{0}^{2}=E / \rho, s=c_{0} t$, and $u=-E v c_{0}^{-1}$. The system is now written as

$$
\begin{aligned}
u_{s} & =\sigma_{x}, \\
E \varepsilon_{s} & =u_{x} .
\end{aligned}
$$

Since there will be no room for confusion in what follows, we proceed to rename the scaled time by $t$.

We fit the nonlinear part of the function $\sigma_{\mathrm{p}}(\varepsilon)$, as given in Fig. 10, by means of a parabola. We write $\varepsilon=\widetilde{H}\left(\sigma_{\mathrm{p}}(\varepsilon)\right)$, where $\widetilde{H}(\sigma)$ is defined by

$$
\widetilde{H}(\sigma)= \begin{cases}E^{-1} \sigma & \text { if } \sigma=Y, \\ E^{-1} \sigma+E^{-1} \beta(\sigma-Y)^{2} & \text { if } \sigma \geq Y,\end{cases}
$$

with $\beta$ a parameter to be assigned. Then, along a deformation, we have the stressstrain relation:

$$
\varepsilon= \begin{cases}E^{-1} \sigma+E^{-1} \beta\left(\sigma_{\max }-Y\right)^{2} & \text { if } \sigma<\sigma_{\max }, \\ \widetilde{H}(\sigma) & \text { if } \sigma=\sigma_{\max },\end{cases}
$$

where $\sigma_{\max }=\sigma_{\mathrm{p}}\left(\varepsilon_{\max }\right)$. We write $H(\sigma)=\beta(\sigma-Y)^{2}$. Now system (28) is expressed

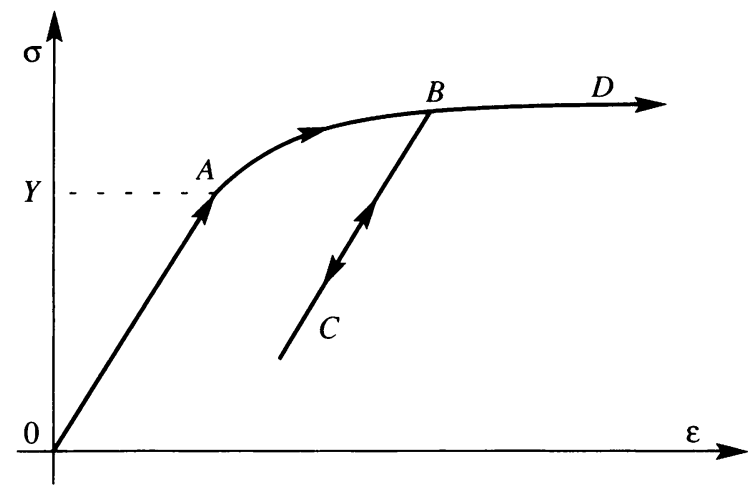

FIG. 10. Stress-strain curve 
as

$$
\begin{aligned}
& u_{t}=\sigma_{x}, \\
& (\sigma+H(\gamma))_{t}=u_{x}, \\
& \gamma=\max \{\sigma(x, s) \text { for } s \leq t\},
\end{aligned}
$$

which follows the description provided in the previous sections.

In Lee's analysis we expect to observe an elastic linear loading wave attached to the leading edge of a plastic rarefaction propagating from the left boundary. When the elastic wave reaches the right boundary, it reflects as an unloading wave which dampens the plastic wave. From Lee's analysis, in the $(x, t)$-plane we expect regions of loading and unloading to appear roughly as in Fig. 11.

Lee pointed out that at a critical value of the velocity at the left boundary, the upper "bag" of plastic deformation will detach from the main body. This situation is observed in our calculations. We fix the parameter $\beta, \beta=0.5$ and look at solutions for different left boundary conditions. We performed two experiments with velocity $v_{L}=-6$ and -4.2 respectively. In Fig. 12, we show the regions of plastic deformation and the contour plots of the stress and yield stress. The first graph corresponds to the case where $v_{L}=-6$ and we observe that the plastic region is simply connected. The second graph has values $v_{L}=-4.2$ and corresponds to the case where the elastic region splits into two pieces.

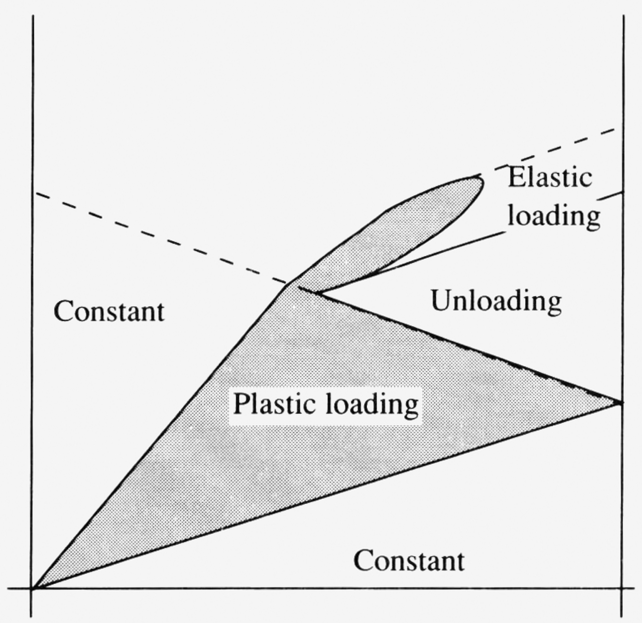

FIG. 11. Loading regions 

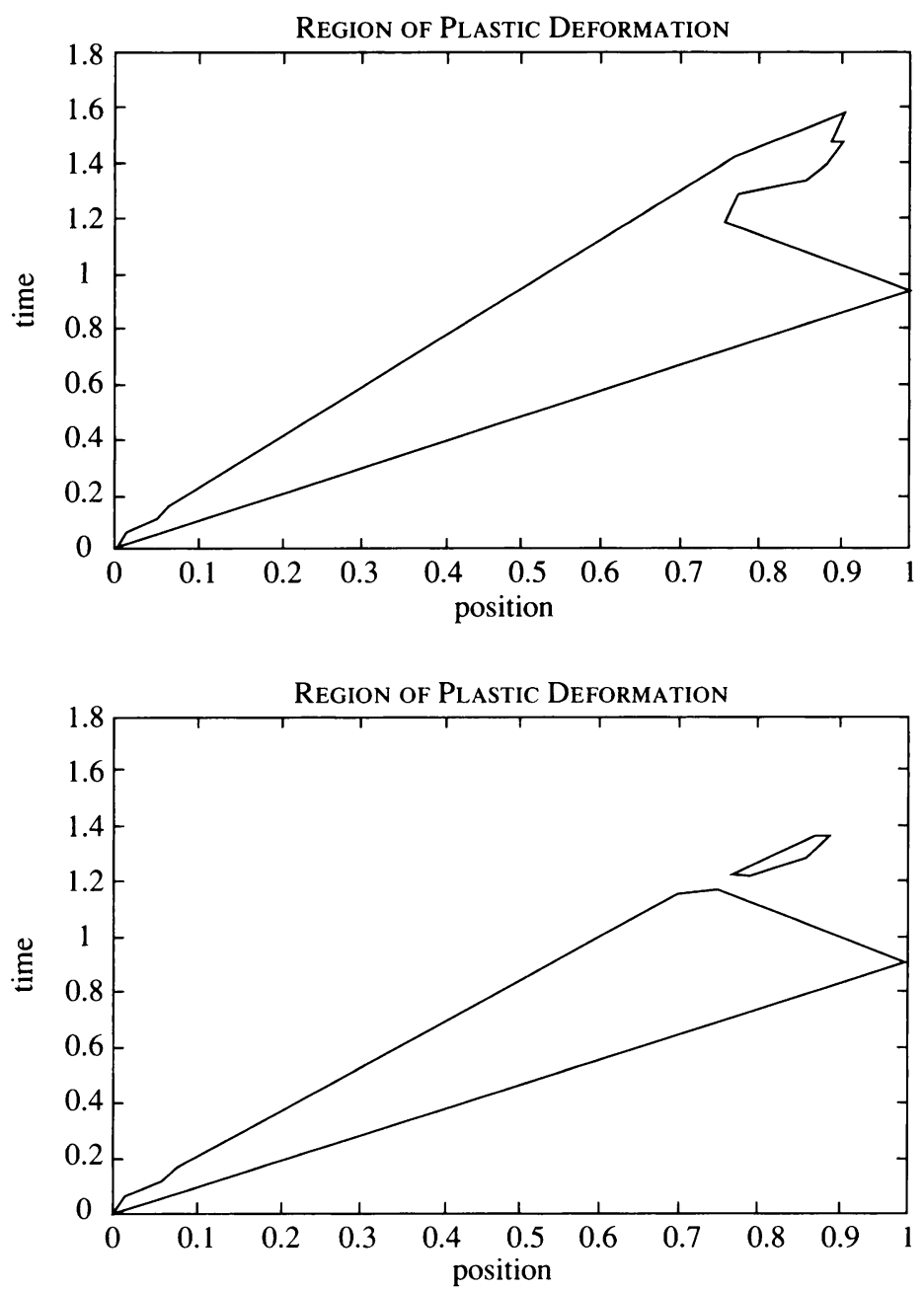

FIG. 12. Regions of plastic deformation in two of Lee's examples

9. Appendix: Wave curves. The function $\varphi(\sigma)$, as defined in (15), is a monotone increasing function while defined. Therefore, a sufficient condition for the existence of a global solution to the Riemann problem is that $\varphi(\sigma)$ be an unbounded function.

We examine the growth of $\varphi$ in various cases.

1. Assume that $\alpha=0$. In this case $\gamma=\sigma$ when loading and

$$
d \varphi=c\left(1+H^{\prime}(\sigma)\right)^{1 / 2} d \sigma .
$$

-(a) If there is a $\sigma_{\mathrm{P}}<+\infty$ such that $\lim _{\sigma \rightarrow \sigma_{\mathrm{P}}} H^{\prime}(\sigma)=\infty$, near $\sigma_{\mathrm{P}}$ we write $H^{\prime}(\sigma)=O\left(\left(\sigma_{\mathrm{p}}-\sigma\right)^{-\beta}\right)$.

(a1) If $\beta \geq 2$ then $\lim _{\sigma \rightarrow \sigma_{\mathrm{p}}} \varphi(\sigma)=+\infty$.

(a2) If $\beta<2$ then $\lim _{\sigma \rightarrow \sigma_{\mathrm{p}}} \varphi(\sigma)$ is bounded.

-(b) Otherwise $\varphi(\sigma)$ is defined for all $\sigma$ and concave up, therefore unbounded. 
2. For $\alpha>0$.

$\bullet$ (a) If there is a $\gamma_{\mathrm{P}}<+\infty$ such that $\lim _{\gamma \rightarrow \gamma_{\mathrm{P}}} H^{\prime}(\gamma)=\alpha^{-2}$, then $\sigma_{\mathrm{P}}=\sigma\left(\gamma_{\mathrm{P}}\right)$ is also finite. Near $\gamma_{\mathrm{P}}$, we have that $d \Phi \approx \alpha^{-1}\left(1-\alpha^{2} H^{\prime}(\gamma)\right)^{1 / 2} d \gamma$ and therefore $\varphi(\sigma)=\Phi(\gamma(\sigma))$ is finite as $\sigma \rightarrow \sigma_{\mathrm{P}}$.

-(b) If $\lim _{\gamma \rightarrow \infty} H^{\prime}(\gamma)=\alpha^{-2}$, we write $\left(1-\alpha^{2} H^{\prime}(\gamma)\right)=O\left(\gamma^{-\beta}\right)$ for large $\gamma$, with $\beta<0$.

- If $\beta>2, \varphi(\gamma)$ and $\sigma(\gamma)$ are bounded as $\gamma \rightarrow \infty$.

- If $1<\beta \leq 2, \varphi(\gamma)$ is unbounded and $\sigma(\gamma)$ is bounded as $\gamma \rightarrow \infty$.

- If $\beta \leq 1, \varphi(\gamma)$ and $\sigma(\gamma)$ are bounded as $\gamma \rightarrow \infty$.

$\bullet$ (c) Otherwise $\varphi(\gamma)$ and $\sigma(\gamma)$ are bounded as $\gamma \rightarrow \infty$.

In (3) we give different examples of strain-stress relations. Case (3-i) corresponds to linear plasticity and shear bands cannot be produced. Cases (3-ii) and (3-iii) do allow the growth of a shear band and case (3-iv) does also when $\alpha \neq 0$. We use case (3-iv) with $\alpha=0$ to model the problem studied by Lee [7] for the deformation of a rod. This case does not exhibit shear bands.

Acknowledgments. The author is grateful to D. Schaeffer and M. Shearer for their comments on this work.

\section{REFERENCES}

[1] S. S. Antman and W. G. Szymczak, Nonlinear elasto-plastic waves, Contemp. Math. 100, 27-54 (1989)

[2] I.-L. Chern, J. Glimm, O. McBryan, B. Plohr, and S. Yaniv, Front tracking for gas dynamics, J. Comp. Phys. 62, 83-110 (1986)

[3] A. Chorin, Random choice solutions of hyperbolic systems, J. Comp. Phys. 22, 517-533 (1976)

[4] X. Garaizar and D. Schaeffer, Numerical computations for shear bands in an antiplane shear model, J. Mech. Phys. Solids, Pergamon, Oxford-Elmsford, NY, in press, 1993

[5] J. Glimm, E. Isaacson, D. Marchesin, and O. McBryan, Front tracking for hyperbolic systems, Adv. Appl. Math. 2, 91-119 (1985)

[6] K. Godunov, Finite-difference method for numerical computation of discontinuous solutions of the equations of fluid dynamics, Mat. Sbornik 47, 271-306 (1959)

[7] E. H. Lee, A boundary value problem in the theory of plastic wave propagation, Quart. Appl. Math. 10, 335-346 (1952)

[8] C. Moler and J. Smoller, Elementary interactions in quasilinear hyperbolic systems, Arch. Rat. Mech. Anal. 37, 309-322 (1970)

[9] D. Schaeffer, A mathematical model for localization in granular flow: Postcritical behavior, preprint, 1991

[10] D. G. Schaeffer and Michael Shearer, Scale-invariant initial value problems in one dimensional dynamic elasto-plasticity, with consequences for multidimensional nonassociative plasticity, European Journal of Applied Mathematics (3), 225-254 (1992)

[11] D. G. Schaeffer and Michael Shearer, Private communications, 1992

[12] J. Smoller, Shock-Waves and Reaction-Diffusion Equations Springer-Verlag, New York and Berlin, 1983

[13] T. C. T. Ting, In Propagation of shock waves in solids, E. Varley, editor, pages 41-64, Amer. Soc. Mech. Eng. AMD vol. 17, 1986

[14] John A. Trangenstein and R. B. Pember, Numerical algorithms for strong discontinuities in elasticplastic solids, Journal of Computational Physics, 1991 
[15] B. van Leer, Towards the ultimate conservative difference scheme. $V$. A. second-order sequel to Godunov's method, J. Comp. Phys. 32, 101-136 (1979)

[16] B. van Leer, On the relation between the upwind-difference schemes of Godunov, Engquist-Osher and Roe, SIAM J. Sci. Stat. Comp. 5 (1), 1-20 (1984)

[17] H. C. Yee, On the implementations of a class of upwind schemes for systems of hyperbolic conservation laws, Technical Report TM-86839, NASA, September 1987

[18] H. C. Yee, A class of high-resolution explicit and implicit shock-capturing methods, Technical Report TM 101088, NASA, February 1989 